\title{
DESARROLLO VEGETATIVO DE PATRONES CÍTRICOS CULTIVADOS EN CONDICIONES DE INVERNADERO BAJO DOS SISTEMAS DE RIEGO ${ }^{1}$
}

\author{
GILMAR SCHÄFER ${ }^{2}$, PAULO VITOR DUTRA DE SOUZA³, OTTO CARLOS KOLLER ${ }^{3}$, \\ SÉRGIO FRANCISCO SCHWARZ ${ }^{3}$
}

\begin{abstract}
RESUMEN - En el presente estudio se evaluó el desarrollo vegetativo de patrones cítricos cultivados en invernadero bajo dos sistemas de riego. El experimento se realizó en la Estação Experimental Agronômica de la Universidade Federal do Rio Grande do Sul, ubicada en Eldorado do Sul, Rio Grande do Sul, Brasil, entre los meses de septiembre de 2003 y abril de 2004, totalizando 225 días de experimentación. El diseño experimental fue de parcelas subdivididas, en factorial 2 x 3, con 4 repeticiones de 22 contenedores cada. En las parcelas principales se evaluaron los sistemas de riego (microaspersión y capilaridad) y en las subparcelas los patrones cítricos Poncirus trifoliata (L.) Raf., citrangero 'C37' [P. trifoliata x Citrus sinensis (L.) Osb. cv. Pêra] y lima 'Rangpur' (C. limonia Osb.). En condiciones de invernadero los patrones cítricos presentan un desarrollo vegetativo más rápido bajo riego por capilaridad respecto a la microaspersión. Los patrones cítricos evaluados presentan desarrollos vegetativos distintos, donde el citrangero 'C37' supera a los demás.
\end{abstract}

Términos para indexación: Citrus; sistemas de riego, cultivo protegido, propagación.

\section{VEGETATIVE DEVELOPMENT OF CITRUS SEEDLINGS CULTIVATED AT GREENHOUSE CONDITIONS AND SUBMITTED TO TWO IRRIGATIONS SYSTEMS}

\begin{abstract}
The aim of the present work was to evaluate the vegetative development of citrus rootstock seedlings cultivated under greenhouse conditions with two irrigation systems. The experiment was conducted at the Estação Experimental Agronômica, Universidade Federal do Rio Grande do Sul, located in Eldorado do Sul, Rio Grande do Sul, Brazil, from September 2003 to April 2004, totalizing 225 days of experimentation. The experimental design was a split-plot, in a $2 \times 3$ factorial, with 4 replications of 22 pots each. In the main plot the irrigation systems was evaluated (micro sprinkler and capillarity) and in the split-plot the citrus rootstocks [Trifoliate orange - Poncirus trifoliata (L.) Raf., 'C37' citrange - P. trifoliata x Citrus sinensis (L.) Osb. cv. Pêra and 'Rangpur' lime - C. limonia Osb.] were evaluated. The main result showed in conditions of greenhouse citrus rootstock seedlings presented a great vegetative development under irrigation by capillarity in relation to the micro sprinkler. The evaluated citrus rootstock seedling display different vegetative developments, where the ' $\mathrm{C} 37$ ' citrange was more vigorous.
\end{abstract}

Index terms: Citrus sp., irrigation systems, greenhouse, propagation.

\section{INTRODUCCIÓN}

En la producción de plantones cítricos en Rio Grande do Sul más de un $90 \%$ de los injertos son sobre Poncirus trifoliata (Schäfer, 2000). La falta de diversificación, en los patrones, torna esta citricultura vulnerable frente a las nuevas enfermedades.

En los últimos años, tras el aumento del número de huertos y viveros infectados por Xantomonas axonopodis pv. citri y tras el aparecimiento de enfermedades causadas por Xilella fastidiosa, además de las enfermedades de suelo, el sistema de producción de plantones cítricos en Brasil está cambiando para el cultivo en invernaderos. Este sistema, ya empleado en otros países, dificulta la ocurrencia de enfermedades, por el aislamiento del semillero y del vivero, empleo de sustratos y agua de calidad.

En la primera etapa para la obtención de estas plantas se utilizan, con grandes ventajas, semilleros móviles (bandejas de espuma de poliestireno o contenedores de forma cónica), mantenidos bajo invernaderos. A continuación, la formación de los plantones se completa en contenedores o bolsas de plástico de mayor volumen, de donde se obtienen los plantones con hasta doce meses tras la siembra de los patrones (Carvalho \& Souza, 1996).

Además de las ventajas citadas anteriormente, Teófilo Sobrinho (1991) comenta que la siembra en bandejas permite la obtención de plantas con sistemas radiculares de mayor volumen, en consecuencia de la muerte del ápice de la raíz principal, cuando esta alcanza el orificio basal; conlleva a una mayor supervivencia tras el transplante al huerto.

En la fase final de producción del plantón (del injerto hasta la comercialización), en condiciones de invernadero, se emplea de forma más común el sistema de riego por goteo, por su economía de agua, de fertilizantes, además de no mojar la parte aérea del árbol. Sin embargo, en el semillero, donde se emplean contenedores de volumen pequeño, el sistema de riego más común es por microaspersión alta, con un gran consumo de agua y mojando las hojas, favoreciendo el aparecimiento de enfermedades. Sistemas de riego por capilaridad (floating) son más económicos en el consumo de agua, además de no mojar la parte aérea. Este sistema se emplea con suceso en viveros de tabaco y hortalizas (Rodrigues, 2002), presentando potencial de uso en la citricultura.

El objetivo de este estudio es evaluar el desarrollo vegetativo de patrones cítricos cultivados en invernadero bajo dos sistemas de riego, microaspersión y capilaridad.

\section{MATERIAL Y MÉTODOS}

El experimento se realizó en la Estação Experimental Agronômica de la Universidade Federal do Rio Grande do Sul, ubicada en Eldorado do Sul, Rio Grande do Sul, entre los meses de septiembre de 2003 y abril de 2004, totalizando 225 días de experimentación.

El diseño experimental fue de parcelas subdivididas, en factorial $2 \times 3$, con 4 repeticiones de 22 contenedores cada. En las parcelas principales se evaluaron los sistemas de riego (microaspersión y capilaridad) y en las subparcelas los patrones cítricos Poncirus trifoliata (L.) Raf., citrangero ' $\mathrm{C} 37$ ' [P. trifoliata x Citrus sinensis (L.) Osb. cv. Pêra] y lima 'Rangpur' (C. limonia Osb.).

La siembra de los patrones fue en contenedores cónicos de polietileno negro $\left(120 \mathrm{~cm}^{3}\right)$, perforados en la base, a razón de 2 a 3 semillas por contenedor, en sustrato comercial Plantmax Citrus ${ }^{\circledR}$. Tras su emergencia se dejó solamente una planta por contenedor, o sea, aquella más vigorosa. Las características físicas y químicas del sustrato, antes del cultivo, se presentan en la Tabla 6. Para la caracterización química se utilizó la metodología de Tedesco et al. (1995) y para la caracterización física se utilizó la metodología de De Boodt \& Verdonck (1972).

\footnotetext{
${ }^{1}$ (Trabalho 110-2005). Recebido: 08-07-2005. Aceito para publicação: 30-06-2006. Parte da Tese de Doutorado apresentada pelo primeiro autor ao curso de Fitotecnia da Universidade Federal do Rio Grande do Sul. Trabalho realizado com apoio financeiro do CNPq e CAPES.

${ }^{2}$ Eng. Agr., Dr. Rua Pinto Bandeira 1088, CEP 94470-480, Viamão, RS, Brasil. E-mail: gilmarschafer@ hotmail.com.

${ }^{3}$ Eng. Agrônomo, Dr., Prof. Faculdade de Agronomia da UFRGS - Depto. de Horticultura e Silvicultura, Av. Bento Gonçalves 7712, CEP 91501-970, Porto Alegre, RS. Autor para correspondência.E-mail: pvdsouza@ufrgs.br, ockoller@ufrgs.br e schwarz@ufrgs.br.
} 
El sistema de riego por microaspersión se accionó por un temporizador, en intervalos que variaron entre 10 y 30 minutos al día, según la evapotranspiración en el invernadero. El sistema de riego por capilaridad consistió en la inmersión del $60 \%$ del volumen de los contenedores en agua, a partir de su base, por periodos de una hora, dos veces al día. El sistema consistió de la disposición de un filme de polietileno negro por debajo de los contenedores de manera a formar un tanque para contención del agua. Tal tanque se rellenó por medio de un motor de lavadora conectado a un depósito de agua de $500 \mathrm{~L}$, accionado por un temporizador. Cumplido el tiempo de riego programado, otro motor regresaba el agua al depósito de $500 \mathrm{~L}$ en un sistema circulante.

El abonado se hizo por la aplicación de $0,1 \mathrm{~g}$ planta de $\mathrm{N}$ a los 117 y 180 días de la siembra, tras su dilución en $5 \mathrm{~mL}$ de agua por planta en cada aplicación (Vichiato et al., 1998).

Se evaluaron el porcentaje de contenedores en los cuales emergió por lo menos una planta (PCEUP); vigor: diámetro del cuello $(\mathrm{mm})$; longitud de la parte aérea (medida del cuello hasta el ápice, en $\mathrm{cm})$; área foliar por planta $\left(\mathrm{cm}^{2}\right)$; materia seca de raíces, parte aérea y total (raíces + parte aérea) (g); contenido nutricional foliar: $\mathrm{N}$ total, $\mathrm{P}$, $\mathrm{K}, \mathrm{Ca}, \mathrm{Mg}$.

El área foliar se midió por medio de un medidor foliar Marca LI-Cor, modelo LI-3100.

Para determinación de la masa de las plantas se secaron en estufa a $65^{\circ} \mathrm{C}$ hasta valor constante.

Los contenidos nutricionales foliares se determinaron según metodología descrita por Tedesco et al. (1995), en el Laboratório de Solos e Tecidos del Departamento de Solos de la Universidade Federal do Rio Grande do Sul.

A los resultados se les aplicó el análisis de la varianza, utilizando el test de Duncan $(\mathrm{P}<0,05)$ para la separación de medias. Al porcentaje de contenedores en los cuales emergió por lo menos una planta y a la longitud de la parte aérea se les aplicó el análisis de regresión. Además, se hizo un análisis de correlación entre las variables observadas.

\section{RESULTADOS Y DISCUSIÓN}

No se encontró interacción entre los factores riego y patrones para el porcentaje de contenedores en los cuales emergió por lo menos una planta (Tabla 1).

El porcentaje de contenedores en los cuales emergió por lo menos una planta no se afectó por los sistemas de riego, solamente se encontraron diferencias entre los patrones (Tabla 1). El $P$. trifoliata presentó una germinación inferior a los demás. Estos resultados están de acuerdo con Davies \& Albrigo (1994), que indican que la germinación de las semillas de cítricos varían con la temperatura ( 9 a $38^{\circ} \mathrm{C}$ ), humedad del aire y cultivar, sin depender de la luminosidad. A partir de estas informaciones, se puede inferir que la humedad del sustrato fue suficiente para una buena germinación de las semillas en los dos sistemas de riego en estudio. A su vez, aunque se encontraron diferencias en la germinación de los patrones, eso no fue limitante, pues la germinación fue mayor al $90 \%$ en todos los casos.

El diámetro del cuello de los patrones fue semejante entre los sistemas de riego (Tabla 1), puesto que en esta etapa del desarrollo ocurre un incremento más fuerte en longitud y en el área foliar. El citrangero ' $\mathrm{C} 37$ ' presentó un mayor diámetro del cuello entre los patrones en estudio, seguido por el $P$. trifoliata y por la lima 'Rangpur' (Tabla 1). El diámetro del cuello se relaciona directamente con el diámetro a 10-20 cm más arriba del mismo (punto donde se realza el injerto), por eso es una característica importante, pues determina el momento del injerto, o sea, un adelanto en el diámetro significa una reducción en el período de producción del plantón. Sin embargo, para que se pudiera cuantificar la reducción del tiempo para el injerto, los patrones deberían cultivarse hasta el momento exacto de la operación de injerto, lo que no se objetivó en este estudio. La longitud (Tabla 2), el área foliar (Tabla 3) y la materia seca total de la planta o de la parte aérea (Tabla 4) fue mayor en aquellas que se cultivaron bajo el sistema de riego por capilaridad. Las diferencias en la longitud de las plantas variaron el 22\% para el $P$. trifoliata, el 23,8\% para la lima 'Rangpur' y
TABLA 1 - Influencia de dos sistemas de riego sobre el porcentual de contenedores de los cuales emergió al menos una planta (PCEUP) y el diámetro del cuello de patrones cítricos cultivados en invernadero bajo dos sistemas de riego. EEA/UFRGS, Eldorado do Sul, 2004.

\begin{tabular}{lccc}
\hline \multicolumn{1}{c}{ Tratamiento } & $\begin{array}{c}\text { PCEUP } \\
(\%)\end{array}$ & $\begin{array}{c}\text { Diámetro } \\
\text { del cuello } \\
(\mathrm{mm})\end{array}$ \\
\hline Sistema de & Capilaridad & 96,2 & 3,40 \\
Riego & Microaspersión & 96,2 & 3,15 \\
\hline Coeficiente de Variación (\%) & 1,8 & 4,19 \\
\hline \hline \multirow{2}{*}{ Patrón } & 'C37'2 & $97,7 \mathrm{a}^{1}$ & $3,90 \mathrm{a}^{\mathrm{I}}$ \\
& P. trifoliata & $91,5 \mathrm{~b}$ & $3,12 \mathrm{~b}$ \\
& 'Rangpur' & $99,4 \mathrm{a}$ & $2,80 \mathrm{c}$ \\
\hline \multicolumn{2}{l}{ Coeficiente de Variación (\%) } & 4,8 & 8,83 \\
\hline
\end{tabular}

${ }^{\mathrm{T}}$ Medias seguidas por letras distintas, en la columna, indican diferencias estadísticamente significativas por el test de Duncan $(P<0,05)$. ${ }^{2}$ Patrón cítrico desarrollado en el Centro de Pesquisa de Fruticultura de Taquari-RS, Brasil. (FEPAGRO)

\section{el 64\% para el citrangero 'C37', en favor del riego por capilaridad.}

Con relación a la longitud de los patrones hubo un comportamiento distinto entre los sistemas de riego (Tabla 2). En el sistema por capilaridad, el citrangero 'C37' presentó mayor longitud respecto al $P$. trifoliata y a la lima 'Rangpur'. A su vez, en el sistema por microaspersión, el patrón $P$. trifoliata presentó mayor longitud respecto a los demás. Esta mayor longitud del $P$. trifoliata también se encontró en experimento realizado por Schäfer (2000). Estos resultados indican que el citrangero 'C37', además de tener un mayor vigor respecto al $P$. trifoliata en el campo, también lo tiene en el semillero, cuando el sistema de riego es por capilaridad. Mientras, bajo microaspersión, el $P$. trifoliata presentó mayor longitud, que se explica por la anatomía de sus hojas, o sea, a pesar de haber plantas más grandes en el centro del semillero, por tener hojas más pequeñas permite el paso del agua asta el sustrato en este sistema, lo que no ocurre con los demás patrones que tienen hojas más grandes. En la Figura 1 se observa el incremento linear del crecimiento primario a lo largo del cultivo en los patrones en estudio.

En general, los patrones con mayor desarrollo vegetativo, principalmente en longitud, presentan un mayor número de hojas, lo que conlleva a una mayor área foliar. Entre tanto, la mayor área foliar de los patrones verificada en el riego por capilaridad (Tabla 3) fue consecuencia de hojas más grandes. En la lima 'Rangpur' hubo también un aumento en el número de hojas por planta, generando un incremento del $110 \%$ en el índice de área foliar en este patrón, cuando se comparan los dos sistemas de riego.

El área foliar por planta, número y área foliar por hoja (Tabla 3) fueron significativamente distintas, relacionándose con las características fenotípicas de cada patrón en estudio. La lima 'Rangpur' presentó hojas más grandes con respecto a los demás. El citrangero 'C37' presentó hojas más grandes que el $P$. trifoliata, por ser un híbrido de este con naranjo dulce [Citrus sinensis (L.) Osb.].

La materia seca de las raíces (Tabla 4) presentó un comportamiento inverso a los demás parámetros de desarrollo vegetativo evaluados para el efecto de los sistemas de riego. Las plantas que se cultivaron bajo el sistema de riego por microaspersión presentaron una mayor cantidad de materia seca de las raíces en

TABLA 2 - Longitud de patrones cítricos cultivados en invernadero bajo dos sistemas de riego. EEA/UFRGS, Eldorado do Sul, 2004.

\begin{tabular}{lccc}
\hline \multirow{2}{*}{\multicolumn{1}{c}{ Sistema de riego }} & \multicolumn{3}{c}{ Longitud de la planta (cm) } \\
\cline { 2 - 4 } & 'Rangpur' & 'C37' & $\begin{array}{c}\text { 'C. } \\
\text { trifoliata }\end{array}$ \\
\cline { 2 - 4 } & C 26,82 a & A 34,44 a & B 31,92 a \\
\hline Capilaridad & B 21,66 b & B 20,98 b & A 26,17 b \\
Microaspersión & & 4,95 \\
\hline C.V.(\%)-Sistema de Riego & & 4,77 \\
C.V. (\%)-Patrón & & \\
\hline
\end{tabular}

${ }^{1}$ Medias seguidas por letras distintas, minúsculas en la columna y mayúsculas en la línea, indican diferencias estadísticamente significativas por el test de Duncan $(P<0,05)$. 
TABLA 3 - Área foliar y número de hojas por planta y, área foliar por hoja, de patrones cítricos cultivados en invernadero bajo dos sistemas de riego. EEA/UFRGS, Eldorado do Sul, 2004.

\begin{tabular}{|c|c|c|c|c|c|c|c|c|c|}
\hline \multirow{3}{*}{ Sistema de riego } & \multicolumn{3}{|c|}{ Área foliar $\left(\mathrm{cm}^{2}\right.$ planta $\left.^{-1}\right)$} & \multicolumn{3}{|c|}{ Número de hojas por planta } & \multicolumn{3}{|c|}{ Área foliar por hoja $\left(\mathrm{cm}^{2}\right.$ hoja $\left.{ }^{-1}\right)$} \\
\hline & \multicolumn{3}{|c|}{ Patrón } & \multicolumn{3}{|c|}{ Patrón } & \multicolumn{3}{|c|}{ Patrón } \\
\hline & 'C37' & P. trifoliata & 'Rangpur' & 'C37' & P. trifoliata & 'Rangpur' & 'C37' & P. trifoliata & 'Rangpur' \\
\hline Capilaridad & B 97,9 a & C 32,8 a & A 159,6 a & B $17,2 \mathrm{a}^{1}$ & B 19,7 a & A 27,1 a & A 5,7 a & B 1,67 a & A 5,95 a \\
\hline Microaspersión & B $47,7 \mathrm{~b}$ & C $22,1 \mathrm{~b}$ & A $75,20 \mathrm{~b}$ & B 14,8 a & A 22,0 a & A $20,7 \mathrm{~b}$ & A $3,2 \mathrm{~b}$ & B $1,04 \mathrm{~b}$ & A $3,63 \mathrm{~b}$ \\
\hline \multirow{2}{*}{\multicolumn{2}{|c|}{$\begin{array}{l}\text { C.V. }(\%) \text { - Sistema } \\
\text { C.V. }(\%) \text { - Patrón }\end{array}$}} & 1,27 & & & 3,93 & & & 6,93 & \\
\hline & & 3,91 & & & 13,53 & & & 9,36 & \\
\hline
\end{tabular}

${ }^{1}$ Medias seguidas por letras distintas, minúsculas en la columna y mayúsculas en la línea, indican diferencias estadísticamente significativas por el test de Duncan $(P<0,05)$

comparación a las que se cultivaron con el sistema de riego por capilaridad. Se esperaba que esta característica tuviera el mismo comportamiento de las demás; sin embargo esta respuesta se puede explicar por dos factores: primeramente, las plantas que se cultivaron bajo microaspersión sufrieron poda aérea de sus raíces, lo que no ocurrió con gran efectividad en aquellas con riego por capilaridad. La poda de la raíz principal generó brotación de raíces secundarias, por lo tanto, ocurriendo un aumento en materia seca de las raíces. Por otra parte, el riego por capilaridad puede haber sido más eficiente, disminuyendo la necesidad de desarrollo de las raíces y su búsqueda por agua y nutrientes.

Aunque el desarrollo radicular sea diferenciado, los sistemas de riego generaron más de un $95 \%$ de plantas que formaron turrón en el momento de su remoción del contenedor, lo que es ideal en el repicado.

Las características que se discutieron anteriormente favorecieron a la mayor cantidad de materia seca en el patrón 'C37' (Tabla 4), un $29 \%$ superior a los demás. Esta tendencia fue constante en todas las características de materia seca, con variaciones para el $P$. trifoliata y la lima 'Rangpur', que no difirieron entre si en materia seca total. En valores absolutos, los valores de materia seca encontrados en el presente estudio fueron superiores a los encontrados en otros estudios. Por ejemplo, Schmitz (1998) describe materia seca alrededor del $0,64 \mathrm{~g}^{\text {planta }^{-1}}$ en el patrón $P$. trifoliata y Schäfer (2000) describe materia seca de $0,27 \mathrm{~g} \mathrm{planta}^{-1}$ en el mismo patrón y 0,43 g planta $^{-1}$ para la lima 'Rangpur'.

En Rio Grande do Sul el P. trifoliata presenta mayor utilización cómo patrón respecto a la lima 'Rangpur', principal patrón de la citricultura brasileña, debido principalmente a la inducción de mayor resistencia al frío, menor tamaño de copa y mayor resistencia a enfermedades de suelo. No obstante, su desarrollo vegetativo inicial, en semillero, es superior a los otros patrones (Schäfer, 2000), lo que se observa en la altura de los patrones cultivados bajo microaspersión (Tabla 2). Este mayor desarrollo inicial se explica por el mayor contenido de reservas en sus semillas (semillas más grandes). Sin embargo, tras la necesidad de diversificación y búsqueda de patrones con más vigor en el huerto, el citrangero ' $\mathrm{C} 37$ ' se presenta cómo una alternativa potencial, pues además de la resistencia del $P$. trifoliata, heredó el vigor del naranjo dulce, proporcionando buen desarrollo vegetativo, lo que se observa en este estudio (Tablas 1, 2, 3 y 4).

El mayor desarrollo vegetativo de los patrones cultivados bajo sistema de riego por capilaridad se supone sea por la mejor distribución del agua en este sistema de riego, disminuyendo los daños a la parte aérea por no mojarla, lo que conlleva un mayor desarrollo en longitud y una mayor área foliar (Tabla 3) y, por lo tanto, mayor cantidad de materia seca total (Tabla 4).

Los contenidos en macronutrientes de las hojas se presentan en la Tabla 5. No se encontró interacción significativa entre los sistemas de riego y los patrones para este parámetro de evaluación.

Según los niveles presentados por la Sociedade Brasileira de Ciência do Solo (2004), para plantas productivas, los contenidos en nitrógeno presentes en los patrones se consideran excesivos, en fósforo son satisfactorios a altos, en potasio son excesivos, en calcio son deficientes a bajos y los contenidos en magnesio son bajos.

Con estos resultados se concluye que el abonado realizado con nitrógeno influenció los niveles de éste en la planta, elevándolos excesivamente. Sin embargo, no se constataron síntomas de salinidad en los patrones, lo que encontró Schäfer (2000) al aplicar 0,268 g

TABLA 4 - Materia seca de las raíces, de la parte aérea y materia seca total por planta, de patrones cítricos cultivados en invernadero bajo dos sistemas de riego. EEA/UFRGS, Eldorado do Sul, 2004.

\begin{tabular}{|c|c|c|c|c|}
\hline & Tratamiento & Materia seca de las raíces & Materia seca da la parte aérea & Materia seca total \\
\hline \multirow{2}{*}{ Sist. riego } & Capilaridad & $0,67 b^{1}$ & $1,61 \mathrm{a}$ & $2,27 \mathrm{a}^{1}$ \\
\hline & Microaspersión & $0,82 \mathrm{a}$ & $1,12 \mathrm{~b}$ & $1,94 \mathrm{~b}$ \\
\hline C.V. $(\%)$ & & 5,87 & 7,71 & 6,90 \\
\hline \multirow{3}{*}{ Patrón } & 'C37' & $0,93 \mathrm{a}$ & $1,62 \mathrm{a}$ & $2,56 \mathrm{a}$ \\
\hline & P. trifoliata & $0,68 \mathrm{~b}$ & $1,37 \mathrm{~b}$ & $1,77 \mathrm{~b}$ \\
\hline & 'Rangpur' & $0,62 \mathrm{~b}$ & $1,09 \mathrm{c}$ & $1,99 \mathrm{~b}$ \\
\hline C.V. $(\%)$ & & 19,73 & 14,57 & 15,40 \\
\hline
\end{tabular}
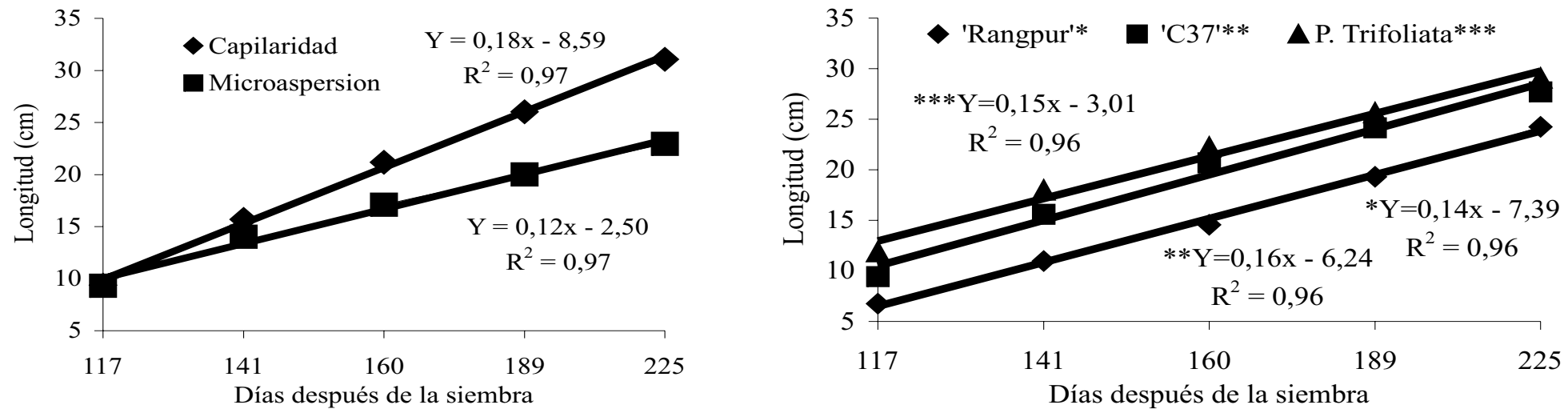

FIGURA 1 - Longitud de patrones cítricos a lo largo de su cultivo en invernadero bajo dos sistemas de riego. EEA/UFRGS, Eldorado do Sul, RS, 2004. 
TABLA 5 - Contenidos de macronutrientes en las hojas de patrones cítricos cultivados en invernadero bajo dos sistemas de riego. EEA/UFRGS, Eldorado do Sul, 2004.

\begin{tabular}{|c|c|c|c|c|c|c|}
\hline \multirow{2}{*}{\multicolumn{2}{|c|}{ Tratamiento }} & \multicolumn{5}{|c|}{ Contenido nutricional de la materia seca total $\left(\mathrm{g} \mathrm{kg}^{-1}\right)$} \\
\hline & & $\mathrm{N}$ & $\mathrm{P}$ & K & $\mathrm{Ca}$ & $\mathrm{Mg}$ \\
\hline \multirow{2}{*}{ Sistema de riego } & Capilaridad & 33,3 & 1,7 & $28,4 \mathrm{a}^{1}$ & $13,7 \mathrm{~b}$ & $2,4 \mathrm{~b}$ \\
\hline & Microaspersión & 33,1 & 1,6 & $22,1 \mathrm{~b}$ & $18,3 \mathrm{a}$ & $2,7 \mathrm{a}$ \\
\hline \multicolumn{2}{|l|}{ C.V. $(\%)$} & 5,3 & 6,0 & 4,5 & 2,2 & 3,3 \\
\hline \multirow{3}{*}{ Patrón } & ‘C37’ & 32,9 & $1,5 \mathrm{c}^{1}$ & $\overline{25,2}$ & $17,6 \mathrm{a}$ & $2,7 \mathrm{a}$ \\
\hline & P. trifoliata & 33,6 & $1,8 \mathrm{a}$ & 25,4 & $16,0 \mathrm{~b}$ & $2,6 \mathrm{a}$ \\
\hline & 'Rangpur' & 33,2 & $1,6 \mathrm{~b}$ & 25,3 & $14,4 \mathrm{c}$ & $2,4 \mathrm{~b}$ \\
\hline \multicolumn{2}{|l|}{ C.V. (\%) } & 9,8 & 4,8 & 7,6 & 6,2 & 8,6 \\
\hline
\end{tabular}

${ }^{1}$ Medias seguidas por letras distintas, en la columna, indican diferencias estadísticamente significativas por el test de Duncan $(P<0,05)$.

TABLA 6 - Contenidos de nutrientes disponibles y características físicas en el sustrato antes del cultivo. EEA/UFRGS, Eldorado do Sul, 2004.

\begin{tabular}{|c|c|c|c|c|c|c|c|c|c|c|c|c|c|c|}
\hline \multirow{2}{*}{ Sustrato } & $\mathrm{K}$ & Al & $\mathrm{Ca}$ & $\mathrm{Mg}$ & $\mathrm{S}$ & $\mathrm{Zn}$ & $\mathrm{Cu}$ & B & Mn & \multirow[t]{2}{*}{ Sólidos } & $\mathrm{EA}^{*}$ & AFD & \multirow[t]{2}{*}{$\mathrm{AT}^{*}$} & $\mathrm{AR}^{*}$ \\
\hline & $--\mathrm{mg} \mathrm{L}^{-1}--$ & & $\operatorname{tol}_{\mathrm{c}}$ & --- & & 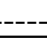 & $\mathrm{ng} \mathrm{L}$ & & & & & & & \\
\hline Citrus $^{\left({ }^{\circledR}\right.}$ & 178 & 0,3 & 34 & 11 & 7,5 & 5,1 & 0,2 & 0,7 & 51 & 18,67 & 32,67 & 9,67 & 3,00 & 36,00 \\
\hline
\end{tabular}

${ }^{*} \mathrm{EA}=$ Espacio de aireación, $\mathrm{AFD}=\mathrm{A} g u a$ fácilmente disponíble, $\mathrm{AT}=\mathrm{A} g u a$ tamponante, $\mathrm{AR}=\mathrm{A} g u a$ Remanescente.

planta $^{-1}$ de $\mathrm{N}$ aplicado en dos veces, resultando en valores de hasta $40,1 \mathrm{~g}$ de nitrógeno por $\mathrm{kg}$ de tejido vegetal.

En los contenidos en nitrógeno y potasio no se encontraron diferencias entre los patrones en estudio. Ya, los contenidos en fósforo, calcio y magnesio variaron según el patrón (Tabla 5). El contenido en fósforo fue mayor en el $P$. trifoliata, seguido por la lima 'Rangpur' y por el 'C37'. El contenido en calcio fue mayor en el 'C37', seguido por el $P$. trifoliata y fue menor en la lima 'Rangpur'. El contenido en magnesio fue semejante en el 'C 37 ' y en el $P$. trifoliata, y menor en la lima 'Rangpur'.

Según el análisis químico del sustrato (Tabla 6), se verifica que todos los macronutrientes están presentes en niveles altos, incluso el calcio y el magnesio. Los niveles excesivos de potasio en las hojas, combinados con los bajos niveles de calcio y magnesio, deben ser consecuencia del antagonismo entre éstos y el potasio, pues cuando el potasio está en niveles elevados inhibe la absorción del calcio y del magnesio (Basso et al., 1983; Malavolta \& Violante Neto, 1989).

Las diferencias nutricionales que se encontraron entre los patrones cítricos se deben probablemente a sus características genéticas. Ellas influencian la capacidad de empleo de energía, luz, dióxido de carbono, lo que interfiere en la absorción, en el transporte, en la distribución y también en la interacción entre los nutrientes dentro de las plantas (Gallo et al., 1960; Carvalho \& Souza, 1996).

No se observó efecto del sistema de riego sobre los niveles de nitrógeno y fósforo, sin embargo estos se presentaron en niveles excesivos y altos, respectivamente (Tabla 5).

Los sistemas de riego influyeron en los niveles de potasio, calcio y magnesio. Los niveles de potasio se mostraron menores en las plantas bajo riego por microaspersión, probablemente debido a la mayor pérdida del nutriente por lixiviación. También se verificó el antagonismo entre el potasio y el calcio por una correlación negativa $(r=-0,55$ y probabilidad $>t=0,006)$, en concordancia con lo que se discutió anteriormente. Los contenidos en calcio y en magnesio, aunque distintos estadísticamente, se consideran deficientes y bajos, respectivamente.

Estos resultados demuestran que el abonado en el semillero es crítico y fundamental, y debe ser realizado cuidadosamente. Como ejemplo de esto se verificó que aunque se haya aplicado nitrógeno en pequeñas cantidades, se encontraron niveles excesivos en las hojas. Además, el abonado con altos contenidos en potasio en el sustrato puede afectar la absorción de calcio, conllevando a niveles limitantes en la planta. Estos aspectos fueron citados por Schäfer (2000) y por De Carlos Neto et al. (2002).

\section{CONCLUSIONES}

1) En condiciones de invernadero los patrones cítricos presentan un desarrollo vegetativo más rápido bajo riego por capilaridad respecto a la microaspersión.

2) Los patrones cítricos evaluados presentan desarrollos vegetativos distintos, donde el citrangero 'C 37 ' supera a los demás.

\section{REFERENCIA}

BASSO, C.; MIELNICZUK, J.; BOHNEN, H. Influência da adubação NPK na concentração de nutrientes em folhas de laranjas Valência. Pesquisa Agropecuária Brasileira, Brasília, v. 18, n. 1, p. 17-21, 1983.

CARVALHO, S. A.; SOUZA, M. Doses e freqüência de aplicação de nitrato de potássio no crescimento do limoeiro 'Cravo' e da tangerineira 'Cleópatra' em bandejas. Pesquisa Agropecuária Brasileira, Brasília, v. 31, n. 11, p. 815-822, 1996.

DAVIES, F. S.; ALBRIGO, L. G. Crop production science in horticulture 2: citrus. Wallingford: CAB International, $1994.254 \mathrm{p}$.

DE BOODT, M.; VERDONCK, O. The physical properties of the substrates in horticulture. Acta horticulturae, Wageningen, v. 26, p. 37-44, 1972.

DECARLOS NETO, A.; SIQUEIRA, D. L.;PEREIRA,P.R. G;ALVAREZ, V. H. Crescimento de porta-enxertos de citros em tubetes influenciados por doses de N. Revista Brasileira de Fruticultura, Jaboticabal, v. 24, n. 1, p. 199-203, 2002.

GALLO, J. R.; MOREIRA, S.; RODRIGUEZ, O.; FRAGA JR., C. G. Influência da variedade e do porta-enxerto na composição mineral das folhas de citros. Bragantia, Campinas, v. 19, n. 20, p. 307-318, 1960.

MALAVOLTA, E.; VIOLANTE NETTO, A. Nutrição mineral, calagem, gessagem e adubação dos citros. Piracicaba: Associação Brasileira para Pesquisa da Potassa e do Fosfato, 1989. 153 p.

SCHÄFER, G. Caracterização molecular, diagnóstico e avaliação de porta-enxertos na citricultura gaúcha. $2000.81 \mathrm{f}$. Tesina (Maestría) Programa de Pós-Graduação em Fitotecnia, Faculdade de Agronomia, Universidade Federal do Rio Grande do Sul, Porto Alegre, 2000.

SCHMITZ, J. A. K. Cultivo de Poncirus trifoliata (L.) Raf. em recepientes: influência de substratos e de fungos micorrízicos arbusculares. 1998. 144 f. Tesina (Maestría) Programa de PósGraduação em Fitotecnia, Faculdade de Agronomia, Universidade Federal do Rio Grande do Sul, Porto Alegre, 1998.

SOCIEDADE BRASILEIRA DE CIÊNCIA DO SOLO. Manual de adubação e calagem para os Estados do Rio Grande do Sul e de Santa Catarina. Porto Alegre: SBCS. Núcleo Regional Sul, 2004. $394 \mathrm{p}$.

TEDESCO, M. J.; GIANELLO, C.; BISSANI, C. A.; BOHNEN, H.; VOLKWEISS, S. J. Análises de solo, plantas e outros materiais. 2. ed. Porto Alegre: Departamento de Solos da UFRGS, 1995. 174 p. (Boletim Técnico de Solos, 5).

TEÓFILO SOBRINHO, J. Propagação dos Citros. In: RODRIGUES, O; VIÉGAS, F; POMPEU JUNIOR, J. et al. Citricultura brasileira. Campinas: Fundação Cargill, 1991. v.1, p. 281-301.

VICHIATO, M.; SOUZA, M.; AMARAL, A. M.; MEDEIROS, M. R.; RIBEIRO, W. G. Desenvolvimento e nutrição mineral da tangerineira Cleópatra fertilizada com superfosfato simples e nitrato de amônio em tubetes até a repicagem. Ciência e Agrotecnologia, Lavras, v. 22, n. 1, p. 30-41, 1998. 